\title{
Healing responses following surgical/non-surgical treatment in residual periodontally-involved sites
}

S.K.Y. HO, K.Y. ZEE, and E.F. CORBET, University of Hong Kong, Hong Kong

Objective: The aim of this study was to evaluate the healing responses following surgical or repeated non-surgical treatment in residual pockets of previously non-surgically treated periodontitis patients. Methods: 12 female and 4 male subjects (mean age $=44.5$ years) were recruited for this split-mouth, single-blinded study. Matched-paired-sites with residual probing pocket depth $\geq 6 \mathrm{~mm}$ and bleeding on probing were selected in each patient. Presence of supragingival plaque (PI\%), bleeding on probing (BOP\%), probing pocket depth (PPD) and probing attachment level (PAL) were recorded at baseline and 3 months after treatment. Test sites were selected randomly for repeated non-surgical debridement under local anaesthesia while control sites received surgical treatment. Results: Full-mouth PI\% decreased from $20 \%$ at baseline to $17 \%$ at 3 months and the BOP\% decreased from $25 \%$ to $22 \%$. Mean PPD reduction for test and control sites were $3.3 \mathrm{~mm}$ and $3.1 \mathrm{~mm}$ ( $p>0.05$, paired t-test), mean PAL gains were $2.5 \mathrm{~mm}$ and $1.7 \mathrm{~mm}(\mathrm{p}>0.05)$, and mean increases in recession were $0.8 \mathrm{~mm}$ and $1.4 \mathrm{~mm}$ $(p<0.05)$. Conclusion: The healing responses in residual periodontally-involved sites following non-surgical or surgical treatment were similar and both treatment modalities were effective in PPD reduction and gain of PAL, but surgical debridement caused more recession.

Periodontal Research - Therapy

The Preliminary Program for Annual Scientific Meeting, 19th International Association for Dental Research-Southeast Asia Division and 13th Southeast Asia Association for Dental Education (September 3-6, 2004) 